\title{
APPROXIMATION FOR THE PERCENTILES OF THE SAMPLE COEFFICIENT OF VARIATION
}

\section{NOBUMICHI SHUTOH}

Graduate School of Maritime Sciences

Kobe University

Japan

e-mail: shutoh@maritime.kobe-u.ac.jp

\begin{abstract}
We propose a method of approximation for the percentiles of the sample coefficient of variation based on the samples drawn from a normal population via Cornish-Fisher expansion. We derive the asymptotic expansion of the distribution of the sample coefficient of variation by using the normal approximation of the Chi-square variable and some useful expectation formulas. Finally, we observe the superiority of our result on the percentiles of the test statistic for one-sample problem by conducting Monte Carlo simulation under some selected parameters.
\end{abstract}

\section{Introduction}

In this paper, we consider statistical inference for the coefficient of variation $R$, that is, the ratio of the mean to the standard deviation which has applications in various research areas; see, for example, [7].

Under normality, one of the traditional results in this study is given by [6], who proposed an approximation to the distribution of the sample 
coefficient of variation that asymptotically follows the $\chi^{2}$ distribution (recently, [2] showed that approximation derived by [6] essentially follows a type-II noncentral beta distribution). Several authors concluded that approximation considered in [6] performs well under $R \leq 2 / 3$.

For testing the coefficients of variation, various procedures have been proposed. [1] proposed the likelihood ratio test that follows asymptotically the $\chi^{2}$ distribution under the null hypothesis in $k$-sample problem; this was later modified by [11]. [4] derived the Wald type test and the Score test. [10] considered a divergence based test statistic. Employing the stochastic expansion used in [9], [8] proposed a simple test statistic by using the asymptotic normality of the sample coefficient of variation.

In this paper, we show the asymptotic normality of the sample coefficient of variation. It will be applicable to a test statistic for onesample problem of $R$, which is asymptotically equivalent to [8]. Further, we derive the asymptotic distribution up to the higher order with respect to $n^{-1}$ and $(n-1)^{-1}$, where $n$ denotes the sample size, in order to obtain more accurate approximation for the percentiles of the considered test statistic via Cornish-Fisher expansion. Simulation studies observe the size of the test on the basis of our result, which is compared to the test derived by [8]. Finally, in our concluding remarks, we address the direction for further study.

\section{The Distribution of the Sample Coefficient of Variation}

Let $X_{1}, \ldots, X_{n}$ be the $n$ independent samples from $N\left(\mu, \sigma^{2}\right)$. The sample coefficient of variation $\hat{R}$ is defined as

$$
\hat{R}=\frac{S}{\bar{X}} \stackrel{d}{=} \frac{\sqrt{Y}}{Z+\frac{\sqrt{n}}{R}}, \quad \bar{X}=\frac{1}{n} \sum_{i=1}^{n} X_{i}, \quad S^{2}=\frac{1}{n} \sum_{i=1}^{n}\left(X_{i}-\bar{X}\right)^{2}
$$


where the symbol $\stackrel{d}{=}$ denotes the equivalent in the sense of the distribution, $Y$ is a random variable that follows the $\chi^{2}$ distribution with $n-1$ degrees of freedom, $Z$ is a random variable that follows $N(0,1), Y$ is independent of $Z$, and $R$ is the coefficient of variation $\sigma / \mu$.

Employing the useful notations of the order $n=m \rho, n-1=m \lambda$, where $\rho$ and $\lambda$ are some constants as in [3], and the normalizing approximation of the Chi-square variable

$$
U=\frac{Y-m \lambda}{\sqrt{2 m \lambda}} \stackrel{d}{\rightarrow} N(0,1)(m \rightarrow \infty),
$$

the distribution of the sample coefficient of variation $\hat{R}$ can be expressed as

$$
\hat{R} \stackrel{d}{=} \frac{\sqrt{m \lambda+\sqrt{2 m \lambda} U}}{Z+\frac{\sqrt{m \rho}}{R}}=R \frac{\sqrt{\lambda}}{\sqrt{\rho}}\left(1+\frac{\sqrt{2}}{\sqrt{m \lambda}} U\right)^{\frac{1}{2}}\left(1+\frac{R}{\sqrt{m \rho}} Z\right)^{-1}
$$

Henceforth, we consider the asymptotic distribution of $\widetilde{R}=\sqrt{n /(n-1)} \hat{R}$.

Applying Taylor expansion to the standardized $\widetilde{R}$, we have

$$
T(R)=\frac{\sqrt{m}(\widetilde{R}-R)}{\sigma} \stackrel{d}{=} t_{0}(R)+\frac{t_{1}(R)}{\sqrt{m}}+\frac{t_{2}(R)}{m}+O_{p}\left(m^{-\frac{3}{2}}\right),
$$

where $\sigma=R \sqrt{\{1 /(2 \lambda)\}+\left(R^{2} / \rho\right)}$,

$$
\begin{aligned}
& t_{0}(R)=-\frac{R^{2}}{\sqrt{\rho} \sigma} Z+\frac{R}{\sqrt{2} \sqrt{\lambda} \sigma} U, \\
& t_{1}(R)=\frac{R^{3}}{\rho \sigma} Z^{2}-\frac{R^{2}}{\sqrt{2} \sqrt{\rho \lambda} \sigma} Z U-\frac{R}{4 \lambda \sigma} U^{2}, \\
& t_{2}(R)=-\frac{R^{4}}{\rho \sqrt{\rho} \sigma} Z^{3}+\frac{R^{3}}{\sqrt{2} \rho \sqrt{\lambda} \sigma} Z^{2} U+\frac{R^{2}}{4 \sqrt{\rho} \lambda \sigma} Z U^{2}+\frac{\sqrt{2} R}{8 \lambda \sqrt{\lambda} \sigma} U^{3} .
\end{aligned}
$$


Noting that $T(R) \stackrel{d}{=} t_{0}(R)+o_{p}(1)$, (2) coincides with the result shown by [8].

Theorem 1. For a large $n, \hat{R}$ is asymptotically distributed as $N(R$, $\left.\left\{R^{2} /(n-1)\right\}\left(1 / 2+R^{2}\right)\right)$.

\section{Asymptotic Expansion for the Distribution of the Test Statistic}

In this section, we show the asymptotic expansion of the distribution of $T(R)$ in a similar manner to [5]. Further, we can approximate the percentiles of $T(R)$.

The characteristic function of $T(R)$ can be expanded as

$$
\begin{aligned}
& E[\exp (i t T(R))] \\
& =E\left[\exp \left(i t \cdot t_{0}(R)\right) \cdot \exp \left\{i t\left(\frac{t_{1}(R)}{\sqrt{m}}+\frac{t_{2}(R)}{m}+O_{p}\left(m^{-\frac{3}{2}}\right)\right)\right\}\right] \\
& =E\left[\exp \left(i t \cdot t_{0}(R)\right)\right]+\frac{1}{\sqrt{m}} E\left[-\theta \exp \left(i t \cdot t_{0}(R)\right) t_{1}(R)\right] \\
& \quad+\frac{1}{m} E\left[-\theta \exp \left(i t \cdot t_{0}(R)\right) t_{2}(R)+\frac{\theta^{2}}{2} \exp \left(i t \cdot t_{0}(R)\right)\left\{t_{1}(R)\right\}^{2}\right]+O\left(m^{-\frac{3}{2}}\right),
\end{aligned}
$$

where $\theta=-i t$. In order to obtain the expectations, we have a lemma.

Lemma 2. The density function of $u$ can be obtained as

$$
\begin{aligned}
f_{u}(u)= & \phi(u)\left\{1+\frac{1}{\sqrt{m}}\left(\frac{\sqrt{2}}{3 \sqrt{\lambda}} u^{3}-\frac{\sqrt{2}}{\sqrt{\lambda}} u\right)\right. \\
& \left.+\frac{1}{m}\left(\frac{1}{9 \lambda} u^{6}-\frac{7}{6 \lambda} u^{4}+\frac{2}{\lambda} u^{2}-\frac{1}{6 \lambda}\right)\right\}+O\left(m^{-\frac{3}{2}}\right),
\end{aligned}
$$


where $\phi(z)$ is the density function of $Z$.

Further, let $I_{i}(a)$ be the following integration:

$$
\int_{-\infty}^{\infty} x^{i} \frac{1}{\sqrt{2 \pi}} \exp \left[-\frac{1}{2}(x-(-\theta a))^{2}\right] d x
$$

Then we have

$$
\int_{-\infty}^{\infty} x^{i} \exp (i t \cdot a x) \phi(x) d x=\exp \left(-\frac{1}{2} a^{2} t^{2}\right) I_{i}(a)
$$

To calculate $I_{i}(a)$, we also have the following corollary:

Corollary 3. For $i=0, \ldots, 6$, the integration $I_{i}(a)$ can be obtained as

$$
\begin{aligned}
& I_{0}(a)=1, \quad I_{1}(a)=-(\theta a), \quad I_{2}(a)=1+(\theta a)^{2}, \\
& I_{3}(a)=-3(\theta a)-(\theta a)^{3}, \quad I_{4}(a)=3+6(\theta a)^{2}+(\theta a)^{4}, \\
& I_{5}(a)=-15(\theta a)-10(\theta a)^{3}-(\theta a)^{5}, \\
& I_{6}(a)=15+45(\theta a)^{2}+15(\theta a)^{4}+(\theta a)^{6} .
\end{aligned}
$$

By using Lemma 2 and Corollary 3, we have the characteristic function stated in (3) and the distribution function of $T(R)$.

Theorem 4. The distribution function of $T(R)$ is obtained as

$$
\begin{aligned}
\Phi(x)+ & {\left[a_{11}(R)+a_{12}(R) H_{2}(x)\right.} \\
+ & \left.a_{21}(R) H_{1}(x)+a_{22}(R) H_{3}(x)+a_{23}(R) H_{5}(x)\right] \phi(x)+O_{\frac{3}{2}},
\end{aligned}
$$

where $\mathrm{O}_{\frac{3}{2}}$ denotes the remainder terms of the $3 / 2$-th order with respect to $n^{-1}$ and $(n-1)^{-1}$, 
60

$$
\begin{aligned}
c^{\frac{1}{2}} a_{11}(R) & =\frac{1}{4(n-1)}-\frac{R^{2}}{n}, \\
c^{\frac{3}{2}} a_{12}(R) & =-\frac{1}{24(n-1)^{2}}-\frac{R^{2}}{2 n(n-1)}-\frac{R^{4}}{n^{2}}, \\
c a_{21}(R)= & \frac{1}{32(n-1)^{2}}-\frac{R^{2}}{4 n(n-1)}-\frac{9 R^{4}}{2 n^{2}}, \\
c^{2} a_{22}(R) & =\frac{1}{96(n-1)^{3}}-\frac{R^{2}}{6 n(n-1)^{2}}-\frac{2 R^{4}}{n^{2}(n-1)}-\frac{4 R^{6}}{n^{3}}, \\
c^{3} a_{23}(R) & =-\frac{1}{1152(n-1)^{4}}-\frac{R^{2}}{48 n(n-1)^{3}}-\frac{R^{4}}{6 n^{2}(n-1)^{2}} \\
& -\frac{R^{6}}{2 n^{3}(n-1)}-\frac{R^{8}}{2 n^{4}}, \\
c= & \frac{1}{2(n-1)}+\frac{R^{2}}{n},
\end{aligned}
$$

and $H_{r}(x)$ denotes the r-th Hermite polynomial:

$$
\begin{aligned}
& H_{1}(x)=x, \quad H_{2}(x)=x^{2}-1, \quad H_{3}(x)=x^{3}-3 x, \\
& H_{5}(x)=x^{5}-10 x^{3}+15 x .
\end{aligned}
$$

Proof. Using Lemma 2 and (4), we obtain the following expressions:

$$
\begin{aligned}
\mathbb{I}_{z}(j) & =E\left[Z^{j} \exp \left(i t \cdot-\frac{R^{2}}{\sqrt{\rho} \sigma} Z\right)\right] \\
& =\int_{-\infty}^{\infty} z^{j} \exp \left(i t \cdot-\frac{R^{2}}{\sqrt{\rho} \sigma} z\right) f_{z}(z) d z \\
& =\exp \left(-\frac{1}{2} \frac{R^{4}}{\rho \sigma^{2}} t^{2}\right) I_{j}\left(-\frac{R^{2}}{\sqrt{\rho} \sigma}\right)
\end{aligned}
$$




$$
\begin{aligned}
\mathbb{I}_{u}(j)= & E\left[U^{j} \exp \left(i t \cdot \frac{R}{\sqrt{2} \sqrt{\lambda} \sigma} U\right)\right] \\
= & \int_{-\infty}^{\infty} u^{j} \exp \left(i t \cdot \frac{R}{\sqrt{2} \sqrt{\lambda} \sigma} u\right) f_{u}(u) d u \\
= & \exp \left(-\frac{1}{2} \frac{R^{2}}{2 \lambda \sigma^{2}} t^{2}\right)\left[I_{j}\left(\frac{R}{\sqrt{2} \sqrt{\lambda} \sigma}\right)\right. \\
& +\frac{1}{\sqrt{m}}\left\{\frac{\sqrt{2}}{3 \sqrt{\lambda}} I_{3+j}\left(\frac{R}{\sqrt{2} \sqrt{\lambda} \sigma}\right)-\frac{\sqrt{2}}{\sqrt{\lambda}} I_{1+j}\left(\frac{R}{\sqrt{2} \sqrt{\lambda} \sigma}\right)\right\} \\
& +\frac{1}{m}\left\{\frac{1}{9 \lambda} I_{6+j}\left(\frac{R}{\sqrt{2} \sqrt{\lambda} \sigma}\right)-\frac{7}{6 \lambda} I_{4+j}\left(\frac{R}{\sqrt{2} \sqrt{\lambda} \sigma}\right)\right. \\
& \left.+\frac{2}{\lambda} I_{2+j}\left(\frac{R}{\sqrt{2} \sqrt{\lambda} \sigma}\right)-\frac{1}{6 \lambda} I_{j}\left(\frac{R}{\sqrt{2} \sqrt{\lambda} \sigma}\right)\right\}+O\left(m^{-\frac{3}{2}}\right) .
\end{aligned}
$$

By using the above expressions, we derive some moments in order to obtain the main result.

For the leading terms, we need to obtain the expectation $E\left[\exp \left(i t \cdot t_{0}(R)\right)\right]=\mathbb{I}_{z}(0) \mathbb{I}_{u}(0)$ up to the terms of $m^{-1}$. Substituting $j=0$ into (5) and (6) and making use of Corollary 3, we have

$$
\begin{aligned}
\mathbb{I}_{z}(0)= & \exp \left(-\frac{1}{2} \frac{R^{4}}{\rho \sigma^{2}} t^{2}\right), \\
\mathbb{I}_{u}(0)= & \exp \left(-\frac{1}{2} \frac{R^{2}}{2 \lambda \sigma^{2}} t^{2}\right)\left\{1+\frac{1}{\sqrt{m}}\left(-\frac{\theta^{3} R^{3}}{6 \lambda^{2} \sigma^{3}}\right)\right. \\
& \left.+\frac{1}{m}\left(\frac{\theta^{4} R^{4}}{8 \lambda^{3} \sigma^{4}}+\frac{\theta^{6} R^{6}}{72 \lambda^{4} \sigma^{6}}\right)\right\}+O\left(m^{-\frac{3}{2}}\right) .
\end{aligned}
$$

Because it holds that

$$
\exp \left(-\frac{1}{2} t^{2}\right)=\exp \left(-\frac{1}{2} \frac{R^{4}}{\rho \sigma^{2}} t^{2}\right) \exp \left(-\frac{1}{2} \frac{R^{2}}{2 \lambda \sigma^{2}} t^{2}\right)
$$


we can obtain

$$
\begin{aligned}
E\left[\exp \left(i t \cdot t_{0}(R)\right)\right]= & \exp \left(-\frac{1}{2} t^{2}\right)\left\{1+\frac{1}{\sqrt{m}}\left(-\frac{\theta^{3} R^{3}}{6 \lambda^{2} \sigma^{3}}\right)\right. \\
& \left.+\frac{1}{m}\left(\frac{\theta^{4} R^{4}}{8 \lambda^{3} \sigma^{4}}+\frac{\theta^{6} R^{6}}{72 \lambda^{4} \sigma^{6}}\right)\right\}+O\left(m^{-\frac{3}{2}}\right) .
\end{aligned}
$$

We also need to obtain the expectation

$$
\begin{aligned}
& E\left[-\theta \exp \left(i t \cdot t_{0}(R)\right) t_{1}(R)\right] \\
& \quad=-\frac{\theta R^{3}}{\rho \sigma} \mathbb{I}_{z}(2) \mathbb{I}_{u}(0)+\frac{\theta R^{2}}{\sqrt{2} \sqrt{\rho \lambda} \sigma} \mathbb{I}_{z}(1) \mathbb{I}_{u}(1)+\frac{\theta R}{4 \lambda \sigma} \mathbb{I}_{z}(0) \mathbb{I}_{u}(2) .
\end{aligned}
$$

Substituting $j=1,2$ into (5) and (6) and making use of Corollary 3, we have

$$
\begin{aligned}
\mathbb{I}_{z}(1)= & \exp \left(-\frac{1}{2} \frac{R^{4}}{\rho \sigma^{2}} t^{2}\right)\left(\frac{\theta R^{2}}{\sqrt{\rho} \sigma}\right), \\
\mathbb{I}_{z}(2)= & \exp \left(-\frac{1}{2} \frac{R^{4}}{\rho \sigma^{2}} t^{2}\right)\left(1+\frac{\theta^{2} R^{4}}{\rho \sigma^{2}}\right) \\
\mathbb{I}_{u}(1)= & \exp \left(-\frac{1}{2} \frac{R^{2}}{2 \lambda \sigma^{2}} t^{2}\right)\left\{-\frac{\theta R}{\sqrt{2} \sqrt{\lambda} \sigma}\right. \\
& \left.+\frac{1}{\sqrt{m}}\left(\frac{\theta^{2} R^{2}}{\sqrt{2} \lambda \sqrt{\lambda} \sigma^{2}}+\frac{\theta^{4} R^{4}}{6 \sqrt{2} \lambda^{2} \sqrt{\lambda} \sigma^{4}}\right)\right\}+O\left(m^{-1}\right), \\
\mathbb{I}_{u}(2)= & \exp \left(-\frac{1}{2} \frac{R^{2}}{2 \lambda \sigma^{2}} t^{2}\right)\left\{1+\frac{\theta^{2} R^{2}}{2 \lambda \sigma^{2}}\right. \\
& \left.+\frac{1}{\sqrt{m}}\left(-\frac{2 \theta R}{\lambda \sigma}-\frac{7 \theta^{3} R^{3}}{6 \lambda^{2} \sigma^{3}}-\frac{\theta^{5} R^{5}}{12 \lambda^{3} \sigma^{5}}\right)\right\}+O\left(m^{-1}\right) .
\end{aligned}
$$

Recalling the results of $\mathbb{I}_{z}(0)$ and $\mathbb{I}_{u}(0)$, we can obtain 
$E\left[-\theta \exp \left(i t \cdot t_{0}(R)\right) t_{1}(R)\right]$

$$
\begin{aligned}
= & \exp \left(-\frac{1}{2} t^{2}\right)\left\{\frac{\theta R}{4 \lambda \sigma}-\frac{\theta R^{3}}{\rho \sigma}+\frac{\theta^{3} R^{3}}{8 \lambda^{2} \sigma^{3}}-\frac{\theta^{3} R^{5}}{2 \rho \lambda \sigma^{3}}-\frac{\theta^{3} R^{7}}{\rho^{2} \sigma^{3}}\right. \\
& +\frac{1}{\sqrt{m}}\left(-\frac{\theta^{2} R^{2}}{2 \lambda^{2} \sigma^{2}}-\frac{7 \theta^{4} R^{4}}{24 \lambda^{3} \sigma^{4}}+\frac{2 \theta^{4} R^{6}}{3 \rho \lambda^{2} \sigma^{4}}\right. \\
& \left.\left.-\frac{\theta^{6} R^{6}}{48 \lambda^{4} \sigma^{6}}+\frac{\theta^{6} R^{8}}{12 \rho \lambda^{3} \sigma^{6}}+\frac{\theta^{6} R^{10}}{6 \rho^{2} \lambda^{2} \sigma^{6}}\right)\right\}+O\left(m^{-1}\right) .
\end{aligned}
$$

Finally, we need to obtain the expectation

$$
\begin{aligned}
E[-\theta & \left.\exp \left(i t \cdot t_{0}(R)\right) t_{2}(R)+\frac{\theta^{2}}{2} \exp \left(i t \cdot t_{0}(R)\right)\left\{t_{1}(R)\right\}^{2}\right] \\
= & \frac{\theta R^{4}}{\rho \sqrt{\rho} \sigma} \mathbb{I}_{z}(3) \mathbb{I}_{u}(0)-\frac{\theta R^{3}}{\sqrt{2} \rho \sqrt{\lambda} \sigma} \mathbb{I}_{z}(2) \mathbb{I}_{u}(1)-\frac{\theta R^{2}}{4 \sqrt{\rho} \lambda \sigma} \mathbb{I}_{z}(1) \mathbb{I}_{u}(2) \\
& -\frac{\sqrt{2} \theta R}{8 \lambda \sqrt{\lambda} \sigma} \mathbb{I}_{z}(0) \mathbb{I}_{u}(3)+\frac{\theta^{2} R^{6}}{2 \rho^{2} \sigma^{2}} \mathbb{I}_{z}(4) \mathbb{I}_{u}(0)-\frac{\theta^{2} R^{5}}{\sqrt{2} \rho \sqrt{\rho \lambda} \sigma^{2}} \mathbb{I}_{z}(3) \mathbb{I}_{u}(1) \\
& +\frac{\theta^{2} R^{3}}{4 \sqrt{2} \sqrt{\rho \lambda} \lambda \sigma^{2}} \mathbb{I}_{z}(1) \mathbb{I}_{u}(3)+\frac{\theta^{2} R^{2}}{32 \lambda^{2} \sigma^{2}} \mathbb{I}_{z}(0) \mathbb{I}_{u}(4) .
\end{aligned}
$$

Substituting $j=3,4$ into (5) and (6) and making use of Corollary 3, we have

$$
\begin{aligned}
& \mathbb{I}_{z}(3)=\exp \left(-\frac{1}{2} \frac{R^{4}}{\rho \sigma^{2}} t^{2}\right)\left(\frac{3 \theta R^{2}}{\sqrt{\rho} \sigma}+\frac{\theta^{3} R^{6}}{\rho \sqrt{\rho} \sigma^{3}}\right), \\
& \mathbb{I}_{z}(4)=\exp \left(-\frac{1}{2} \frac{R^{4}}{\rho \sigma^{2}} t^{2}\right)\left(3+\frac{6 \theta^{2} R^{4}}{\rho \sigma^{2}}+\frac{\theta^{4} R^{8}}{\rho^{2} \sigma^{4}}\right), \\
& \mathbb{I}_{u}(3)=\exp \left(-\frac{1}{2} \frac{R^{2}}{2 \lambda \sigma^{2}} t^{2}\right)\left(-\frac{3 \theta R}{\sqrt{2} \sqrt{\lambda} \sigma}-\frac{\theta^{3} R^{3}}{2 \sqrt{2} \lambda \sqrt{\lambda} \sigma^{3}}\right)+O\left(m^{-\frac{1}{2}}\right),
\end{aligned}
$$




$$
\mathbb{I}_{u}(4)=\exp \left(-\frac{1}{2} \frac{R^{2}}{2 \lambda \sigma^{2}} t^{2}\right)\left(3+\frac{3 \theta^{2} R^{2}}{\lambda \sigma^{2}}+\frac{\theta^{4} R^{4}}{4 \lambda^{2} \sigma^{4}}\right)+O\left(m^{-\frac{1}{2}}\right) .
$$

Recalling the results of $\mathbb{I}_{z}(j)$ and $\mathbb{I}_{u}(j)$ for $j=0,1,2$, we can obtain

$$
\begin{aligned}
& E\left[-\theta \exp \left(i t \cdot t_{0}(R)\right) t_{2}(R)+\frac{\theta^{2}}{2} \exp \left(i t \cdot t_{0}(R)\right)\left\{t_{1}(R)\right\}^{2}\right] \\
& =\exp \left(-\frac{1}{2} t^{2}\right)\left(\frac{15 \theta^{2} R^{2}}{32 \lambda^{2} \sigma^{2}}+\frac{\theta^{2} R^{4}}{4 \rho \lambda \sigma^{2}}+\frac{9 \theta^{2} R^{6}}{2 \rho^{2} \sigma^{2}}+\frac{5 \theta^{4} R^{4}}{32 \lambda^{3} \sigma^{4}}-\frac{\theta^{4} R^{6}}{2 \rho \lambda^{2} \sigma^{4}}\right. \\
& \left.+\frac{2 \theta^{4} R^{8}}{\rho^{2} \lambda \sigma^{4}}+\frac{4 \theta^{4} R^{10}}{\rho^{3} \sigma^{4}}+\frac{\theta^{6} R^{6}}{128 \lambda^{4} \sigma^{6}}-\frac{\theta^{6} R^{8}}{16 \rho \lambda^{3} \sigma^{6}}+\frac{\theta^{6} R^{12}}{2 \rho^{3} \lambda \sigma^{6}}+\frac{\theta^{6} R^{14}}{2 \rho^{4} \sigma^{6}}\right) \\
& +O\left(m^{-\frac{1}{2}}\right) .
\end{aligned}
$$

Combining (7)-(9), we have

$$
\begin{aligned}
& \exp \left(-\frac{1}{2} t^{2}\right)\left\{1+\frac{1}{\sqrt{m}}\left(\frac{\theta R}{4 \lambda \sigma}-\frac{\theta R^{3}}{\rho \sigma}-\frac{\theta^{3} R^{3}}{24 \lambda^{2} \sigma^{3}}-\frac{\theta^{3} R^{5}}{2 \rho \lambda \sigma^{3}}-\frac{\theta^{3} R^{7}}{\rho^{2} \sigma^{3}}\right)\right. \\
& +\frac{1}{m}\left(-\frac{\theta^{2} R^{2}}{32 \lambda^{2} \sigma^{2}}+\frac{\theta^{2} R^{4}}{4 \rho \lambda \sigma^{2}}+\frac{9 \theta^{2} R^{6}}{2 \rho^{2} \sigma^{2}}-\frac{\theta^{4} R^{4}}{96 \lambda^{3} \sigma^{4}}+\frac{\theta^{4} R^{6}}{6 \rho \lambda^{2} \sigma^{4}}+\frac{2 \theta^{4} R^{8}}{\rho^{2} \lambda \sigma^{4}}+\frac{4 \theta^{4} R^{10}}{\rho^{3} \sigma^{4}}\right. \\
& \left.\left.+\frac{\theta^{6} R^{6}}{1152 \lambda^{4} \sigma^{6}}+\frac{\theta^{6} R^{8}}{48 \rho \lambda^{3} \sigma^{6}}+\frac{\theta^{6} R^{10}}{6 \rho^{2} \lambda^{2} \sigma^{6}}+\frac{\theta^{6} R^{12}}{2 \rho^{3} \lambda \sigma^{6}}+\frac{\theta^{6} R^{14}}{2 \rho^{4} \sigma^{6}}\right)\right\}+O\left(m^{-\frac{3}{2}}\right) .
\end{aligned}
$$

Inverting the characteristic function, the distribution function can be obtained as

$$
\begin{aligned}
\Phi(x) & +\frac{1}{\sqrt{m}}\left\{\frac{R}{4 \lambda \sigma}-\frac{R^{3}}{\rho \sigma}-\left(\frac{R^{3}}{24 \lambda^{2} \sigma^{3}}+\frac{R^{5}}{2 \rho \lambda \sigma^{3}}+\frac{R^{7}}{\rho^{2} \sigma^{3}}\right) H_{2}(x)\right\} \phi(x) \\
& +\frac{1}{m}\left\{\left(\frac{R^{2}}{32 \lambda^{2} \sigma^{2}}-\frac{R^{4}}{4 \rho \lambda \sigma^{2}}-\frac{9 R^{6}}{2 \rho^{2} \sigma^{2}}\right) H_{1}(x)\right.
\end{aligned}
$$




$$
\begin{aligned}
& +\left(\frac{R^{4}}{96 \lambda^{3} \sigma^{4}}-\frac{R^{6}}{6 \rho \lambda^{2} \sigma^{4}}-\frac{2 R^{8}}{\rho^{2} \lambda \sigma^{4}}-\frac{4 R^{10}}{\rho^{3} \sigma^{4}}\right) H_{3}(x) \\
& \left.-\left(\frac{R^{6}}{1152 \lambda^{4} \sigma^{6}}+\frac{R^{8}}{48 \rho \lambda^{3} \sigma^{6}}+\frac{R^{10}}{6 \rho^{2} \lambda^{2} \sigma^{6}}+\frac{R^{12}}{2 \rho^{3} \lambda \sigma^{6}}+\frac{R^{14}}{2 \rho^{4} \sigma^{6}}\right) H_{5}(x)\right\} \phi(x) \\
& +O\left(m^{-\frac{3}{2}}\right),
\end{aligned}
$$

which completes the proof.

By the last theorem, we have approximation to the upper $100 \alpha$ percentile of $T(R)$, i.e., $t(R, \alpha)$ that satisfies $\operatorname{Pr}[T(R)>t(R, \alpha)] \simeq \alpha$ can be obtained as

$$
t(R, \alpha)=z_{\alpha}+F_{1}\left(R, z_{\alpha}\right)+F_{2}\left(R, z_{\alpha}\right),
$$

where $z_{\alpha}$ is the upper $100 \alpha$ percentile of the standard normal distribution,

$$
\begin{aligned}
F_{1}(R, x)= & -a_{11}(R)-a_{12}(R) H_{2}(x), \\
F_{2}(R, x)= & -\frac{1}{2} a_{11}(R)\left[a_{11}(R)-4 a_{12}(R)\right] x \\
& -a_{12}(R)\left[a_{11}(R)-2 a_{12}(R)\right] x H_{2}(x) \\
& -\frac{1}{2}\left\{a_{12}(R)\right\}^{2} x\left\{H_{2}(x)\right\}^{2}-a_{21}(R) H_{1}(x) \\
& -a_{22}(R) H_{3}(x)-a_{23}(R) H_{5}(x) .
\end{aligned}
$$

\section{Simulation Studies}

In order to investigate the performance of the testing procedure based on $T(R)$ and $t(R, \alpha)$ for one-sample problem, we conduct a Monte Carlo simulation with 1,000,000 replications under the selected parameters: $\alpha=0.10,0.05, n=10,15,20,40, R=0.3,0.6$. We primarily evaluate 
the empirical size in the following one-sample testing for the coefficient of variation $H_{1}: R=R_{0}, \quad H_{2}: R \leq R_{0}$, and $H_{3}: R \geq R_{0}$. All of the simulation results under $R=R_{0}$ are listed in Tables 4.1-4.3. In this paper, we compare the attained type I error and the nominal type I error denoted by $\alpha$ for the following tests:

- Test 1 ([8]; cf. Theorem 1): The test statistic is

$$
T_{M}\left(R_{0}\right)=\frac{\hat{R}-R_{0}}{R_{0} \sqrt{\frac{1}{2(n-1)}+\frac{R_{0}^{2}}{n-1}}} ;
$$

$H_{1}$ is rejected if $\left|T_{M}\left(R_{0}\right)\right|>z_{\frac{\alpha}{2}}, H_{2}$ is rejected if $T_{M}\left(R_{0}\right)>z_{\alpha}$, and $H_{3}$ is rejected if $T_{M}\left(R_{0}\right)<-z_{\alpha}$.

- Test 2 (Test based on $T(R)$ ): The test statistic is $T\left(R_{0}\right), H_{1}$ is rejected if $\left|T\left(R_{0}\right)\right|>z_{\frac{\alpha}{2}}, H_{2}$ is rejected if $T\left(R_{0}\right)>z_{\alpha}$, and $H_{3}$ is rejected if $T\left(R_{0}\right)<-z_{\alpha}$

- Test 3 (Test based on $T(R)$ with the critical value obtained by Cornish-Fisher expansion up to the terms of $1 / 2$-th order ): The test statistic is $T\left(R_{0}\right), H_{1}$ is rejected if $\left|T\left(R_{0}\right)\right|>t^{*}\left(R_{0}, \alpha / 2\right), H_{2}$ is rejected if $T\left(R_{0}\right)>t^{*}\left(R_{0}, \alpha\right)$, and $H_{3}$ is rejected if $T\left(R_{0}\right)<t^{*}\left(R_{0}, 1-\alpha\right)$, where $t^{*}\left(R_{0}, \alpha\right)=z_{\alpha}+F_{1}\left(R_{0}, z_{\alpha}\right)$

- Test 4 (Test based on $T(R)$ with the critical value obtained by Cornish-Fisher expansion up to the terms of 1st order): The test statistic is $T\left(R_{0}\right), H_{1}$ is rejected if $\left|T\left(R_{0}\right)\right|>t\left(R_{0}, \alpha / 2\right), H_{2}$ is rejected if $T\left(R_{0}\right)>t\left(R_{0}, \alpha\right)$, and $H_{3}$ is rejected if $T\left(R_{0}\right)<t\left(R_{0}, 1-\alpha\right)$. 
For testing $H_{1}$, there are several cases such that Tests 1 and 2 are recommended. In particular, Test 2 is a nearly exact test in all the cases for $R_{0}=0.3$. This implies that the approximated percentiles we proposed are not very useful in these cases. On the other hand, for testing $\mathrm{H}_{2}$ and $\mathrm{H}_{3}$ the tests based on the modified percentiles derived in this paper, i.e., applying Tests 3 and 4 are obviously recommended. In particular, we observe that Test 4 can almost exactly control type I error. 
Table 4.1. Empirical size of the test for $H_{1}$

\begin{tabular}{|c|c|c|c|c|}
\hline \multirow[b]{2}{*}{$n$} & \multicolumn{4}{|c|}{$R_{0}=0.3$ and $\alpha=0.10$} \\
\hline & Test 1 & Test 2 & Test 3 & Test 4 \\
\hline 10 & 0.088799 & 0.096023 & 0.087964 & 0.089321 \\
\hline 15 & 0.092960 & 0.097974 & 0.090986 & 0.091797 \\
\hline 20 & 0.094837 & 0.098067 & 0.091989 & 0.092481 \\
\hline \multirow[t]{2}{*}{40} & 0.097217 & 0.099045 & 0.094441 & 0.094689 \\
\hline & \multicolumn{4}{|c|}{$R_{0}=0.6$ and $\alpha=0.10$} \\
\hline$n$ & Test 1 & Test 2 & Test 3 & Test 4 \\
\hline 10 & 0.083176 & 0.101864 & 0.064327 & 0.057172 \\
\hline 15 & 0.088983 & 0.101481 & 0.067334 & 0.061362 \\
\hline 20 & 0.091760 & 0.101129 & 0.069853 & 0.064910 \\
\hline \multirow[t]{2}{*}{40} & 0.095809 & 0.100495 & 0.076505 & 0.073543 \\
\hline & \multicolumn{4}{|c|}{$R_{0}=0.3$ and $\alpha=0.05$} \\
\hline$n$ & Test 1 & Test 2 & Test 3 & Test 4 \\
\hline 10 & 0.035804 & 0.046823 & 0.035433 & 0.035755 \\
\hline 15 & 0.042853 & 0.048058 & 0.038147 & 0.038298 \\
\hline 20 & 0.044876 & 0.048638 & 0.039828 & 0.039911 \\
\hline \multirow[t]{2}{*}{40} & 0.047581 & 0.049608 & 0.043036 & 0.043054 \\
\hline & \multicolumn{4}{|c|}{$R_{0}=0.6$ and $\alpha=0.05$} \\
\hline$n$ & Test 1 & Test 2 & Test 3 & Test 4 \\
\hline 10 & 0.042427 & 0.058993 & 0.032642 & 0.027590 \\
\hline 15 & 0.044780 & 0.056288 & 0.031283 & 0.027172 \\
\hline 20 & 0.045895 & 0.054776 & 0.031242 & 0.027636 \\
\hline 40 & 0.048238 & 0.052736 & 0.033449 & 0.031012 \\
\hline
\end{tabular}


Table 4.2. Empirical size of the test for $\mathrm{H}_{2}$

\begin{tabular}{|c|c|c|c|c|}
\hline \multirow[b]{2}{*}{$n$} & \multicolumn{4}{|c|}{$R_{0}=0.3$ and $\alpha=0.10$} \\
\hline & Test 1 & Test 2 & Test 3 & Test 4 \\
\hline 10 & 0.059291 & 0.094065 & 0.098529 & 0.099800 \\
\hline 15 & 0.066672 & 0.095718 & 0.099156 & 0.099948 \\
\hline 20 & 0.070467 & 0.095653 & 0.098684 & 0.099268 \\
\hline \multirow[t]{2}{*}{40} & 0.079379 & 0.097370 & 0.099361 & 0.099629 \\
\hline & \multicolumn{4}{|c|}{$R_{0}=0.6$ and $\alpha=0.10$} \\
\hline$n$ & Test 1 & Test 2 & Test 3 & Test 4 \\
\hline 10 & 0.086272 & 0.119602 & 0.104625 & 0.101295 \\
\hline 15 & 0.089378 & 0.116410 & 0.102808 & 0.100319 \\
\hline 20 & 0.090970 & 0.114373 & 0.102117 & 0.100141 \\
\hline \multirow[t]{2}{*}{40} & 0.094155 & 0.110297 & 0.100897 & 0.099811 \\
\hline & \multicolumn{4}{|c|}{$R_{0}=0.3$ and $\alpha=0.05$} \\
\hline$n$ & Test 1 & Test 2 & Test 3 & Test 4 \\
\hline 10 & 0.030765 & 0.052833 & 0.049489 & 0.050066 \\
\hline 15 & 0.034387 & 0.052849 & 0.049719 & 0.050092 \\
\hline 20 & 0.036585 & 0.052142 & 0.049425 & 0.049647 \\
\hline \multirow[t]{2}{*}{40} & 0.040605 & 0.051766 & 0.049609 & 0.049733 \\
\hline & \multicolumn{4}{|c|}{$R_{0}=0.6$ and $\alpha=0.05$} \\
\hline$n$ & Test 1 & Test 2 & Test 3 & Test 4 \\
\hline 10 & 0.054028 & 0.077903 & 0.056929 & 0.052126 \\
\hline 15 & 0.054084 & 0.073017 & 0.054641 & 0.051053 \\
\hline 20 & 0.053825 & 0.069762 & 0.053378 & 0.050604 \\
\hline 40 & 0.053519 & 0.064110 & 0.051818 & 0.050254 \\
\hline
\end{tabular}


Table 4.3. Empirical size of the test for $\mathrm{H}_{3}$

\begin{tabular}{|c|c|c|c|c|}
\hline \multirow[b]{2}{*}{$n$} & \multicolumn{4}{|c|}{$R_{0}=0.3$ and $\alpha=0.10$} \\
\hline & Test 1 & Test 2 & Test 3 & Test 4 \\
\hline 10 & 0.136235 & 0.104466 & 0.097801 & 0.099521 \\
\hline 15 & 0.130466 & 0.103482 & 0.098891 & 0.099904 \\
\hline 20 & 0.126678 & 0.102754 & 0.099014 & 0.099718 \\
\hline \multirow[t]{2}{*}{40} & 0.119087 & 0.101731 & 0.099316 & 0.099631 \\
\hline & \multicolumn{4}{|c|}{$R_{0}=0.6$ and $\alpha=0.10$} \\
\hline$n$ & Test 1 & Test 2 & Test 3 & Test 4 \\
\hline 10 & 0.096378 & 0.078200 & 0.104922 & 0.098339 \\
\hline 15 & 0.099715 & 0.082293 & 0.103866 & 0.099533 \\
\hline 20 & 0.100487 & 0.084312 & 0.102722 & 0.099488 \\
\hline \multirow[t]{2}{*}{40} & 0.101434 & 0.088586 & 0.101260 & 0.099630 \\
\hline & \multicolumn{4}{|c|}{$R_{0}=0.3$ and $\alpha=0.05$} \\
\hline$n$ & Test 1 & Test 2 & Test 3 & Test 4 \\
\hline 10 & 0.058034 & 0.043190 & 0.048243 & 0.049116 \\
\hline 15 & 0.058573 & 0.045125 & 0.049268 & 0.049734 \\
\hline 20 & 0.058252 & 0.045925 & 0.049546 & 0.049921 \\
\hline \multirow[t]{2}{*}{40} & 0.056612 & 0.047279 & 0.049794 & 0.049933 \\
\hline & \multicolumn{4}{|c|}{$R_{0}=0.6$ and $\alpha=0.05$} \\
\hline$n$ & Test 1 & Test 2 & Test 3 & Test 4 \\
\hline 10 & 0.029148 & 0.023961 & 0.058551 & 0.046692 \\
\hline 15 & 0.034889 & 0.028464 & 0.055862 & 0.048403 \\
\hline 20 & 0.037935 & 0.031367 & 0.054452 & 0.049027 \\
\hline 40 & 0.042290 & 0.036385 & 0.051962 & 0.049422 \\
\hline
\end{tabular}




\section{Conclusion and Discussion}

We can approximate the percentiles of the sample coefficient of variation via the normal approximation of the Chi-square variable and Cornish-Fisher expansion. We also present the direction to derive the expectation in order to obtain the main result in this paper.

By the simulation results, in particular, the tests based on the approximate percentiles are recommended for the use of the one-sided test in many cases: it is obviously implied that our approximation performs well when the critical value of the test statistic is not so large. For one-sided test, when the accuracy of the critical value of the test statistic is strongly required, we particularly recommend applying Test 4 to the dataset. On the other hand, when the simplicity of the critical value is favorable, we recommend applying Test 2 or Test 3 .

Finally, we address future problems. A similar theoretical result may also be derived on the basis of Wilson and Hilferty's transformation instead of (1). In this case, its effect appears in the terms of the higher order and it may improve our results. In the case that Test 3 is not conservative and Test 4 is conservative, the correction of $t_{1}\left(R_{0}, \alpha\right)$ and $t_{0}\left(R_{0}, \alpha\right)$ by the other tuning parameter will be considered in order to obtain more accurate percentiles in a view of the practical application.

\section{References}

[1] B. M. Bennett, On an approximate test for homogeneity of coefficients of variation, In: Walter John Ziegler Contributing to Applied Statistics Birkhauser Verlag Basel and Stuttgart (1976), 169-171.

[2] J. Forkman and S. Verrill, The distribution of McKay's approximation for the coefficient of variation, Statistics \& Probability Letters 78 (2008), 10-14.

[3] Y. Fujikoshi and T. Seo, Asymptotic approximations for EPMC's of the linear and the quadratic discriminant functions when the sample sizes and the dimension are large, Random Operators and Stochastic Equations 6 (1998), 242-269.

[4] R. C. Gupta and S. Ma, Testing the equality of coefficients of variation in $k$ normal populations, Communications in Statistics-Theory and Methods 25 (1996), 115-132. 
[5] C. Matsumoto, An optimal discriminant rule in the class of linear and quadratic discriminant functions for large dimension and samples, Hiroshima Mathematical Journal 34 (2004), 231-250.

[6] A. T. McKay, Distribution of the coefficient of variation and the extended $t$-distribution, Journal of Royal Statistical Society 95 (1932), 695-698.

[7] E. G. Miller and M. J. Karson, Testing equality of two coefficients of variation, American Statistical Association: Proceedings of the Business and Economics Section Part I (1977), 278-283.

[8] E. G. Miller, Asymptotic test statistics for coefficients of variation, Communications in Statistics-Theory and Methods 20 (1991), 3351-3363.

[9] D. G. Nel, On the symmetric multivariate normal distribution and the asymptotic expansion of a Wishart matrix, South African Statistical Journal 12 (1978), 145-159.

[10] M. C. Pardo and J. A. Pardo, Use of Rényi's divergence to test for the equality of the coefficients of variation, Journal of Computational and Applied Mathematics 116 (2000), 93-104.

[11] N. J. Shafer and J. A. Sullivan, A simulation study of a test for the equality of the coefficients of variation, Communications in Statistics-Simulation and Computation 15 (1986), 681-695. 